\title{
Update on Bladder, Kidney, and Prostate Cancer from the European Society for Medical Oncology (ESMO) Annual Meeting 2016
}

\section{Anil Kapoor, MD, FRCSC}

Professor of Surgery (Urology), McMaster University, Associate Editor (Oncology), Canadian Urological Association Journal (CUAJ), Hamilton, ON, Canada

Cite as: Can Urol Assoc J 2016;10(11-12Suppl6):S223. http://dx.doi.org/10.5489/cuaj.4280

$\mathrm{T}$ he 2016 Annual Meeting of the European Society for Medical Oncology (ESMO) took place in Copenhagen, Denmark, October 7-11, 2016. This year's ESMO meeting included a broad spectrum of new research across a range of different malignancies. New data on the efficacy of immuno-oncology agents (nivolumab, pembrolizumab, and atezolizumab) in bladder cancer has revolutionized the treatment of advanced bladder cancer (CHECKMATE, KEYNOTE, and IMvigor). In the kidney cancer field, for the first time we have a positive adjuvant treatment result for those patients at high risk for recurrence post-tumour resection (S-TRAC). We also have a new option with cabozantinib in the first-line treatment of metastatic kidney cancer (CABOSUN). In the prostate cancer field, the long-term results of CHAARTED in the low-volume disease patients are summarized, demonstrating only the high-volume disease patients benefited. The final negative results of the AFFINITY trial are also reported, as well as data on the role on pembrolizumab in pretreated prostate cancer patients.

Overall, this is a promising time with new options for patients with genitourinary malignancies. We have summarized the most clinically important research presented in the fields of bladder cancer, kidney cancer, and prostate cancer. We hope you find this report informative.

Acknowledgement: The authors would like to thank STA Healthcare Communications for editorial assistance with this report.

Correspondence: Dr. Anil Kapoor, McMaster University, Hamilton, 0N, Canada; akapoor@mcmaster.ca 